
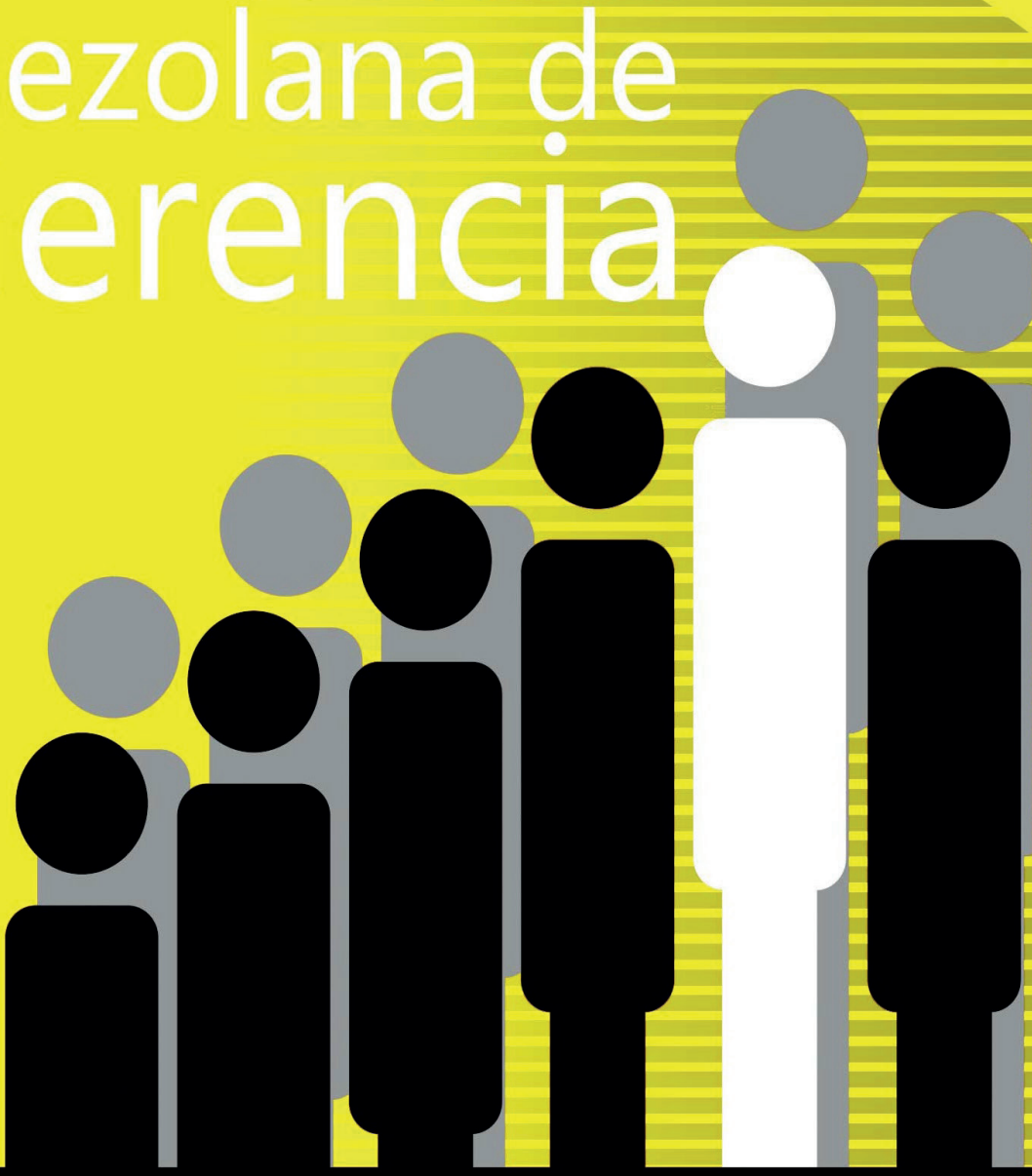


\title{
Minería del cobre en Perú: análisis de las variables exógenas y endógenas para gestionar su desarrollo
}

\author{
Andújar-Palao, José Miguel* \\ Ormachea-Hermoza, Rodrigo ${ }^{*}$ \\ Ruiz-Ruiz, Marcos Fernando** \\ Chirinos-Cuadros, Carlos Ricardo
}

\section{Resumen}

A pesar de tener una economía emergente, Perú es uno de los principales productores cupríferos del mundo, sosteniendo gran parte de su desarrollo sobre la base de esta industria. En tal sentido, el objetivo de esta investigación fue analizar el sistema industrial de la minería de cobre en Perú a partir de sus variables exógenas y endógenas. Con ayuda de un diseño exploratorio y descriptivo, se realizó un análisis estructural (técnica de la prospectiva estratégica francesa) con la participación de un panel de 20 expertos conocedores de la industria minera y de la herramienta Matriz de Impactos Cruzados y Multiplicación Aplicada a una Clasificación. Los resultados identificaron como variables clave al atractivo de inversión, a los conflictos socioambientales y al manejo político; los cuales representan el carácter exógeno de la industria cuprífera peruana. Variables endógenas al sistema, como la eficiencia productiva, la logística del transporte, la generación de empleos y el suministro de energía; se caracterizaron como exclusivamente dependientes de la evolución de las variables exógenas mencionadas. Este estudio constituye un punto de partida importante para la gestión de posibles escenarios futuros de la minería de cobre en Perú; así como para la inversión en su desarrollo económico, social y ambiental a nivel local y regional.

Palabras clave: Cobre; inversión minera; conflictos socioambientales; política minera; Perú.

Recibido: 20.10.20 Aceptado: 18.02.21

Investigador en proyectos mineros, con grado en Ingeniería Industrial por la Universidad de Lima. Email: im.andujarp@hotmail.com ORCID: https://orcid.org/0000-0001-7226-1857

* Investigador en proyectos mineros, con grado en Ingeniería Industrial por la Universidad de Lima. Email: rodrigo.ormache@gmail.com ORCID: https://orcid.org/0000-0001-9571-3174

*** Doctor en Ciencias de la Educación e Ingeniero Industrial. Profesor de proyectos y coordinador de investigación en la Carrera de Ingeniería Industrial de la Universidad de Lima. Consultor en temas de prospectiva estratégica social y análisis estructural. Email (autor para correspondencia): mruiz@ulima.edu.pe ORCID: https://orcid.org/0000-0001-5147-8512

*** Magíster en Administración e Ingeniero Industrial. Profesor de proyectos e investigador de la Carrera de Ingeniería Industrial de la Universidad de Lima. Consultor y académico con amplia experiencia en el diseño y evaluación de proyectos de inversión. Email: cchirino@ulima.edu.pe ORCID: https://orcid.org/0000-0001$\underline{9061-3131}$ 


\title{
Copper mining in Peru: analysis of exogenous and endogenous variables to manage its development
}

\begin{abstract}
Despite having an emerging economy, Peru is one of the main copper producers in the world, sustaining much of its development on the basis of this industry. In this sense, the objective of this research was to analyze the industrial system of copper mining in Peru from its exogenous and endogenous variables. With the help of an exploratory and descriptive design, a structural analysis (French strategic foresight technique) was carried out with the participation of a panel of 20 experts familiar with the mining industry and the MICMAC tool (Matrix of Crossed Impacts and Multiplication Applied to a Classification). The results identified as key variables the attractiveness of investment, socio-environmental conflicts and political management; which represent the exogenous character of the Peruvian copper industry. Variables endogenous to the system, such as productive efficiency, transport logistics, job creation and energy supply; they were characterized as exclusively dependent on the evolution of the aforementioned exogenous variables. This study constitutes an important starting point for the management of possible future scenarios for copper mining in Peru; as well as for investment in its economic, social and environmental development at the local and regional level.
\end{abstract}

Key words: copper; mining investment; socio-environmental conflicts; mining policy; Peru

\section{Introducción}

A lo largo de los años, la industria minera ha contribuido a mantener el equilibrio macroeconómico de un país emergente como Perú (Nugra et al, 2021). Desde el año 2019 Perú es el segundo productor más grande de cobre, representando el $12 \%$ de la producción mundial con aproximadamente 2,46 millones de toneladas métricas (García y Pantigoso, 2020); siendo superado únicamente por Chile con el $28 \%$ de la producción mundial (Servicio Geológico de los Estados Unidos [USGS], 2020). Además, hay que tener en cuenta que
Perú, junto con Australia, es uno de los países con las mayores reservas mundiales de cobre; alcanzando entre ambos los 87 millones de toneladas métricas (USGS, 2020). Asimismo, el $14,8 \%$ del territorio peruano se encuentra bajo concesiones mineras, de las cuales, solo han sido explotadas el 0,9\% (Gonzales et al, 2019). Hoy en día existen 25 nuevos proyectos cupríferos en Perú que representan el $52 \%$ de la cartera de proyectos de construcción de mina del 2019 y el $71 \%$ de las inversiones (Dirección General de Promoción y Sostenibilidad Minera [DGPSM], 2020). 
Al estudiar la industria minera cuprífera peruana como un sistema que debe ser gestionado para su desarrollo económico, socioambiental y político a nivel local y regional; resulta imprescindible identificar y analizar cuáles son aquellas variables exógenas y endógenas que lo caracterizan y cómo se relacionan entre sí. Este análisis estructural permitirá tomar decisiones, tanto a nivelgubernamental como privado, para conducir dicho sistema hacia escenarios futuros más promisorios. No obstante, tal análisis debe partir de una mirada contextualizada de la industria del cobre en Perú que permita construir el inventario de todas sus variables constitutivas. Para tal fin, este estudio empleó el análisis estructural, una metodología que se basa en la reflexión colectiva de un grupo de expertos para describir y analizar un sistema a partir de la relación de todas las variables que lo caracterizan (Godet, 2007; Ruiz-Ruiz, 2017).

El diseño propuesto para esta investigación tuvo tres etapas: la construcción del inventario de variables para la industria del cobre en Perú, el análisis de la relación entre ellas y, finalmente, el análisis de sus variables constituidas como clave. La investigación se efectuó con la ayuda de 20 expertos del sector minero peruano. El proceso metodológico seguido consistió en identificar las relaciones de influencia y dependencia entre todas las variables exógenas y endógenas para determinar aquellas que son más relevantes (variables clave). Fue similar a la metodología Delphi, y se complementó con algunos análisis estadísticos generales (medidas de tendencia central y de dispersión) para el procesamiento sucesivo de las respuestas que permitieran alcanzar consensos entre los expertos y la validación final de la estructura del sistema (García y Suárez, 2013).

\section{Contextualización de la industria del cobre en Perú}

En esta sección, se realiza un análisis de los elementos que forman parte del contexto de la industria del Cobre en Perú, considerando factores económicos, empresariales, sociales, ambientales, políticos y tecnológicos.

\subsection{Contexto económico y empresarial}

Perú resulta ser el país con el costo de producción de cobre más competitivo a nivel mundial, con un valor de 0,85 US $\$$ / libra; razón por la cual atrae y viabiliza la inversión minera. Paralelamente, el país andino posee inmejorables condiciones geológicas y procura implementar las mejores prácticas mineras en cuanto a producción, gestión de gastos administrativos, eficiencia en el uso de energía, combustible y flete (Belapatiño et al, 2020). Estos costos, incluso, podrían ser mejorados empleando un suministro de energía renovable que disminuya su valor actual de 60-100 Euros/MW-h a 30-55 Euros/MW-h para el año 2050 (Hass et al, 2020).

En el sector minero peruano el transporte representa el $44 \%$ del costo logístico, porcentaje muy elevado en comparación con el $8 \%$ de Estados Unidos. Esto se debe, principalmente, al estado actual de la infraestructura vial de Perú: los caminos secundarios y terciarios no están diseñados para permitir un tránsito fluido. El $90 \%$ de las vías rurales y departamentales se encuentran sin asfaltar, dificultando el transporte de vehículos pesados. Esta 
realidad obliga a reducir la eficiencia del transporte urbano y del transporte en general. Un ejemplo de esta desatención es la inversión del gobierno de 800 millones de dólares para la modernización exclusiva del puerto del Callao desatendiendo la inversión en otras vías de acceso que podrían evitar ineficiencias que causan sobrecostos logísticos (Cornejo, 2018). GahonaFlores (2020) señala que la cadena de suministro juega un rol clave al nivel estratégico de las empresas mineras, por lo cual esta ineficiencia logística es una desventaja de Perú frente a otros países mineros.

A pesar de todo, la actividad minera siempre ha impulsado el desarrollo económico peruano. De acuerdo con el Ministerio de Energía y Minas de Perú (Minem, 2019), la minería aportó el 10\% del PBI peruano en el 2018; del cual, el $50 \%$ corresponde exclusivamente al cobre. Esto se debe, en términos de volumen, a que se trata del mineral de mayor relevancia para la economía local, manteniendo un nivel de participación del $54 \%$ en el total de las exportaciones nacionales mineras.

Según una proyección del Instituto Geológico, Minero y Metalúrgico de Perú (Ingemmet, 2017), el sector minero generaría un ingreso a la nación de 758000 millones de dólares para el año 2050, de los cuales, el $69 \%$ correspondería exclusivamente al cobre. Un estudio del Organismo Supervisor de la Inversión en Energía y Minería Osinergmin (2017), determinó que el impacto de la industria del cobre en la economía peruana es tal que, un incremento en su precio de $19 \%$ produciría un aumento de $1,28 \%$ en el PBI y un incremento de su producción de $28 \%$ produciría una subida del $\mathrm{PBI}$ de $1,35 \%$. Por este motivo, la volatilidad de los precios de los minerales afecta directamente a las empresas mineras que dependen de las condiciones del mercado; es decir, ante un periodo de recesión o estancamiento de la economía mundial, los precios de los minerales bajan y por consiguiente las exportaciones mineras también (Gomero, 2017).

Las fluctuaciones del precio de los minerales explican el índice de endeudamiento de las empresas mineras peruanas durante el periodo 2006-2016 (Cárcamo, 2018). Cabe resaltar, además, que las actividades mineras en el Perú son estimuladas también por la inversión privada, siendo una variable determinante para proyectar el crecimiento anual esperado. En tal sentido, la minería representa el $13 \%$ de la inversión privada en Perú, con un valor de 25191 millones de dólares entre los años 2015-2019 según la Sociedad Nacional de Minería, Petróleo y Energía (SNMPE, 2020). Incluso, dicha inversión registró un crecimiento de $26 \%$ durante el periodo 2017 a 2018, alcanzando el monto de 4947 millones de dólares (El Peruano, 2019). El año 2019 la inversión privada mantuvo su ritmo de fuerte expansión -aunque con algo de desaceleración- creciendo un 18\% (Belapatiño et al, 2020). Para el 2020, se proyectó una inversión de alrededor de 6300 millones de dólares, identificando también un crecimiento de mucho menor ritmo respecto al año anterior; alcanzando, a pesar de la coyuntura de estricto confinamiento por pandemia, solo un 9\% (Gestión, 2020).

Finalmente, Perú es uno de los grandes proveedores de cobre para la creciente demanda de China, destinando el $67 \%$ de sus exportaciones a dicho país (DGPSM, 2020). El país asiático es el mayor demandante de cobre del mundo, 
consumiendo anualmente el $50 \%$ de la producción mundial (10 millones de toneladas métricas en el año 2019); por lo cual, su poder de negociación como cliente es determinante para la industria de este mineral (Belapatiño et al, 2020). También se debe tener en cuenta que la transición hacia una economía sostenible genera un incremento en la demanda mundial del cobre. Según expertos como Henckens y Worell (2020), la construcción de una economía sostenible podría requerir diez veces la producción mundial actual de cobre para el año 2050. Sin la transición a este sistema, la demanda solo se triplicaría para el año 2050. Por ejemplo, se estima que la demanda de cobre anual para la producción de vehículos eléctricos en el año 2027 alcanzaría un valor de 1,74 millones de toneladas métricas; es decir, 9,5 veces la demanda del año 2017 (Asociación Internacional de Cobre [ICA], 2017). Asimismo, el crecimiento del mercado de aplicaciones smart home demandará para el año 2030 alrededor de 1,5 millones de toneladas métricas; 39 veces la demanda del año 2018 (ICA, 2019).

\subsection{Contexto social y ambiental}

En el ámbito social, la minería es una gran fuente de empleo para muchos peruanos de la población económicamente activa. Las actividades mineras generaron más de 209 mil empleos en Perú durante el año 2018 y -por cada empleo en minería- se generaron 6,25 empleos más en el resto de la economía. Las actividades de régimen general (correspondientes a proyectos mineros de gran escala) emplearon el $51,6 \%$ de la mano de obra local mientras que, para actividades de pequeños productores mineros, ascendía a 63,58\% (Minem, 2020). Durante la crisis por la pandemia del 2020, la minería permitió evitar el crecimiento del desempleo al no detener sus operaciones (Lominchar, 2020). No obstante, la tasa de informalidad que se observó en diciembre del 2019 fue del $66,4 \%$ según el Instituto Nacional de Estadística e Informática de Perú (INEI, 2020a). Esto debido, principalmente, a una inadecuada fiscalización del Estado (De Echave, 2016). El crecimiento de la minería ilegal se ha extendido en 21 de las 25 regiones del país; principalmente para la extracción del oro (De Echave, 2014).

Por otro lado, según un estudio del Ingemmet (2017), las dos regiones peruanas con mayor potencial de riqueza minera metálica son Cajamarca y Apurímac que, coincidente y paradójicamente, son regiones con los índices de pobreza más altos en Perú (INEI, 2020b). La actividad minera representa el $65 \%$ del total de conflictos socioambientales en el país andino; los cuales se originan, entre otras razones, por la baja atención del Estado, las pugnas distributivas y algunos factores culturales (León, 2019). Rojas de la Puente et al, (2019) consideran que algunas empresas mineras no definen con claridad sus indicadores de impacto social, mientras que Lalama y Bravo (2019) afirman que el cumplimiento de los objetivos de desarrollo sostenible de Perú se ha dificultado por el aumento de problemas de índole social.

La disponibilidad del agua, por ejemplo, es fundamental para las operaciones mineras. Investigadores como González et al, (2019) señalan en sus proyecciones que, en los próximos 10 años, se registrará un decrecimiento en las precipitaciones del sur peruano, 
impactando directamente en los proyectos mineros de cobre. La zona más vulnerable la constituyen regiones sureñas de Perú, como Arequipa y Moquegua; dado que podrían tener dificultades operativas a causa de la escasez del agua. También es preocupante que el $56 \%$ de los nuevos proyectos de la cartera de proyectos de construcción de mina (27 proyectos) cuenten únicamente con una fuente de agua (DGPSM, 2020).

En contextos como Perú, se debe tomar en consideración los factores climáticos y no sólo las catástrofes que ocasionan paros inesperados en la producción minera. Solo de esa forma será posible alcanzar los objetivos de desarrollo sostenible (Rodrigo-Cano et al, 2019).

\subsection{Contexto político}

La privatización del sector minero en Perú genera un marco político particular. El Estado peruano no puede intervenir directamente en las actividades mineras ya que su rol es exclusivamente gestionar los lineamientos y el marco legal a fin de regular la extracción de recursos y promover la investigación. Según Lalama y Bravo (2017), los países con una menor intervención en las actividades mineras han obtenido resultados económicos superiores, incluyendo beneficios sociales. Sin embargo, esto debe ir acompañado de iniciativas del sector privado con una cultura organizacional hacia la responsabilidad social que procure generar empleo para las comunidades.

En un estudio elaborado por BBVA Research (Belapatiño et al, 2020) Perú se encuentra en el puesto 5 del ranking mundial en el índice de aceptación de políticas mineras debiendo mejorar aspectos referidos a la calidad de infraestructura, la regulación laboral, las reclamaciones de tierra bajo disputa, los acuerdos socioeconómicos con las comunidades y la incertidumbre respecto a las áreas protegidas como desiertos. Alvarado Vélez (2018) plantea que las políticas propuestas por el gobierno deben cumplir su propósito no solo a nivel económico sino de acuerdo con el contexto social. Por ello, una economía que depende en gran medida de la minería debería servir para equilibrar los cambios políticos en favor de un mejor tipo de gobernanza.

Por su parte, según la Sociedad Nacional de Minería, Petróleo y Energía de Perú (SNMPE, 2021) el canon minero representa el $50 \%$ de los ingresos obtenidos por el Estado bajo el concepto de impuesto a la renta. Dicho canon es transferido a los gobiernos regionales y locales (municipios provinciales y distritales) por la explotación económica de los recursos mineros.

El $10 \%$ del dinero se entrega a las municipalidades donde se localiza la mina, el $25 \%$ se destina al gobierno local del distrito o provincia de la mina, el $40 \%$ se deriva a las otras provincias y distritos donde se extrae el recurso; y el $25 \%$ restante se entrega al gobierno regional (destinando una porción a las universidades públicas de la región). El principal uso de este recurso debe ser el financiamiento y gestión de proyectos de infraestructura para salud, educación, seguridad, investigación científica y tecnológica; entre otros.

\subsection{Contexto tecnológico}

Desde el punto de vista tecnológico, el uso de la inteligencia artificial y la robótica pueden contribuir a la generación de soluciones para las 
operaciones mineras en los campos de la perforación automatizada, la predicción de esfuerzos y recursos de sostenimiento minero, el control de calidad, entre otros (Schwarz, 2018). Se estima que para los años 2025-2027 los robots representen el $50 \%$ de la fuerza laboral en las minas; permitiendo una reducción de los accidentes mineros en un $75 \%$. Además, se emplearán simuladores para la capacitación de trabajadores en el uso de equipos, mejorando así la seguridad y productividad (Rumbo Minero, 2020). Actualmente, ya existen minas que realizan exploraciones utilizando drones, cámaras GoPro y vehículos autónomos operados de forma remota (Rivera, 2020).

\section{Industria del cobre peruano: variables exógenas y endógenas que la caracterizan y cómo se relacionan}

A partir de una cuidadosa búsqueda documental y un previo análisis retrospectivo teórico del sector minero, que incluyeron los clásicos análisis PEST y de fuerzas de Porter, se propusieron y validaron con algunos de los expertos participantes las 12 variables para el sistema de la minería del cobre de Perú. Dichas variables fueron categorizadas, según su naturaleza, en variables exógenas (Cuadro 1) y variables endógenas (Cuadro 2) para el sistema estudiado. En los cuadros antes mencionados se presenta también una breve descripción y alcance de cada una de dichas variables para su mejor entendimiento.

\section{Cuadro 1 \\ Variables exógenas (externas) para el análisis del sistema minero del cobre en Perú}

\begin{tabular}{|c|c|c|}
\hline Nombre & Código & Descripción y/o alcance \\
\hline Atractivo de inversión & ATIV & $\begin{array}{l}\text { La inversión en el sector minero es clave para el de- } \\
\text { sarrollo del sistema y la producción del cobre, viene } \\
\text { creciendo a medida que surgen nuevas oportunidades } \\
\text { de proyectos mineros }\end{array}$ \\
\hline Conflictos socio-ambientales & COSA & $\begin{array}{l}\text { La gran mayoría de conflictos socio-ambientales en el } \\
\text { Perú se debe a la minería y se originan por baja inter- } \\
\text { vención del gobierno, pugnas distributivas y factores } \\
\text { culturales }\end{array}$ \\
\hline Demanda de China & $\mathrm{DECH}$ & $\begin{array}{l}\text { China consume el } 50 \% \text { de la producción del cobre glo- } \\
\text { bal, por lo cual, las exportaciones de los principales } \\
\text { productores de este mineral, como el Perú, dependen } \\
\text { del consumo del país asiático }\end{array}$ \\
\hline $\begin{array}{l}\text { Demanda de economía sos- } \\
\text { tenible }\end{array}$ & DEES & $\begin{array}{l}\text { La transición hacia una economía sostenible generará } \\
\text { un incremento en la demanda mundial del cobre, como } \\
\text { por ejemplo, la producción de vehículos eléctricos y de } \\
\text { aplicaciones smart home }\end{array}$ \\
\hline
\end{tabular}




\section{Cont... Cuadro 1}

\begin{tabular}{|c|c|c|}
\hline Disponibilidad de agua & DIAG & $\begin{array}{l}\text { El agua es clave para las operaciones mineras, siendo } \\
\text { la geografía, el cambio climático y las precipitaciones, } \\
\text { factores que contribuyen a su disponibilidad }\end{array}$ \\
\hline Manejo político & MAPO & $\begin{array}{l}\text { La minería peruana se enfrenta actualmente a la baja } \\
\text { intervención del gobierno en las mesas de diálogo, a la } \\
\text { complejidad de la burocracia y a la corrupción de los } \\
\text { gobiernos regionales }\end{array}$ \\
\hline Potencial geológico & POGE & $\begin{array}{l}\text { El Perú es el tercer país del mundo con las reservas de } \\
\text { cobre más grandes, sin embargo, dado el alto nivel de } \\
\text { extracción tiene un tiempo de agotamiento estimado } \\
\text { de } 35 \text { años }\end{array}$ \\
\hline Volatilidad del precio & VOPR & $\begin{array}{l}\text { Al ser un commoditie, el precio del cobre depende de } \\
\text { las condiciones del mercado. Ante un periodo de re- } \\
\text { cesión o estancamiento de la economía mundial, los } \\
\text { precios caen junto con las exportaciones }\end{array}$ \\
\hline
\end{tabular}

Fuente: Elaboración propia

\section{Cuadro 2}

\section{Variables endógenas (internas) para el análisis del sistema minero del cobre en Perú}

\begin{tabular}{cll}
\hline Nombre & Código & \multicolumn{1}{c}{ Descripción y/o alcance } \\
\hline $\begin{array}{c}\text { Eficiencia de la producción } \\
\text { Eficiencia logística del trans- } \\
\text { porte }\end{array}$ & EFPR & $\begin{array}{l}\text { El Perú es el país con el menor costo de producción } \\
\text { de cobre debido principalmente a los bajos costos de } \\
\text { mano de obra y de suministro de energía }\end{array}$ \\
\hline $\begin{array}{c}\text { Generación de empleos } \\
\text { Suministro de energía }\end{array}$ & $\begin{array}{l}\text { En el sector minero peruano el transporte tiene un cos- } \\
\text { to más elevado que otros países debido a la compleji- } \\
\text { dad geográfica del Perú y la falta de inversión pública }\end{array}$ \\
\hline GEEM & $\begin{array}{l}\text { Las actividades mineras generan encadenamientos } \\
\text { productivos en las comunidades cercanas, contribu- } \\
\text { yendo a la creación de puestos de trabajo y estabilidad } \\
\text { laboral }\end{array}$ \\
\hline SUEN & $\begin{array}{l}\text { El Perú, dada sus condiciones geológicas, tiene la } \\
\text { oportunidad de migrar a un suministro de energía re- } \\
\text { novable en los próximos años }\end{array}$ \\
\hline
\end{tabular}

Fuente: Elaboración propia 


\subsection{Relación estructural entre las variables: ¿cuáles son clave?}

Las variables anteriormente presentadas, fueron sometidas a la consideración de los 20 expertos participantes y calificadas en rondas sucesivas en una matriz relacional y estructural (Godet y Durance, 2011) a fin de identificar el grado de relación entre ellas. Los expertos se dividieron en siete áreas de especialidad: 1 experto en asuntos ambientales, 3 expertos en comercialización de minerales, 2 expertos en suministro de energía, 5 expertos en logística minera, 1 experto en mantenimiento, 3 expertos de operaciones en minas y 2 expertos en proveedores del sistema (maquinaria pesada y explosivos); además contamos, para efectos de la validación de resultados, con el apoyo de 3 especialistas de reconocida trayectoria e influencia en el sistema minero de Perú.

Para esta etapa se consideró además, dentro del perfil de los expertos participantes, que cuenten con no menos de 20 años de experiencia en el sector minero peruano, que tengan comprobado dominio en un área específica relacionada al contexto del tema de estudio, que ocupen puestos gerenciales de alto nivel (directores ejecutivos, presidencias, gerentes regionales y gerentes de división) y que hayan trabajado en las principales mineras peruanas, empresas privadas o instituciones públicas vinculadas al sector.

En la matriz para el análisis relacional y estructural, se calificaron los niveles de motricidad y dependencia que determinan las coordenadas de cada variable en un plano cartesiano de influencias (ordenadas) y dependencias (abscisas). Para la identificación de aquellas variables más importantes del sistema, se diagramó la matriz de influencia indirecta (MII) cuyo objetivo es identificar las relaciones entre todas las variables que, por su acción indirecta, juegan también un rol clave en el sistema de acuerdo a la teoría prospectiva (Godet y Durance, 2011). Se identificaron como variables clave del sistema al atractivo de inversión (ATIV), a los conflictos socio-ambientales (COSA) y al manejo político (MAPO); todas ellas categorizadas anteriormente como variables externas al sistema estudiado. El Gráfico 1 muestra la matriz de influencias directas e indirectas entre las variables del sistema, con sus respectivas zonas de análisis. 


\section{Gráfico 1}

\section{Matriz de influencias entre las variables del sistema}

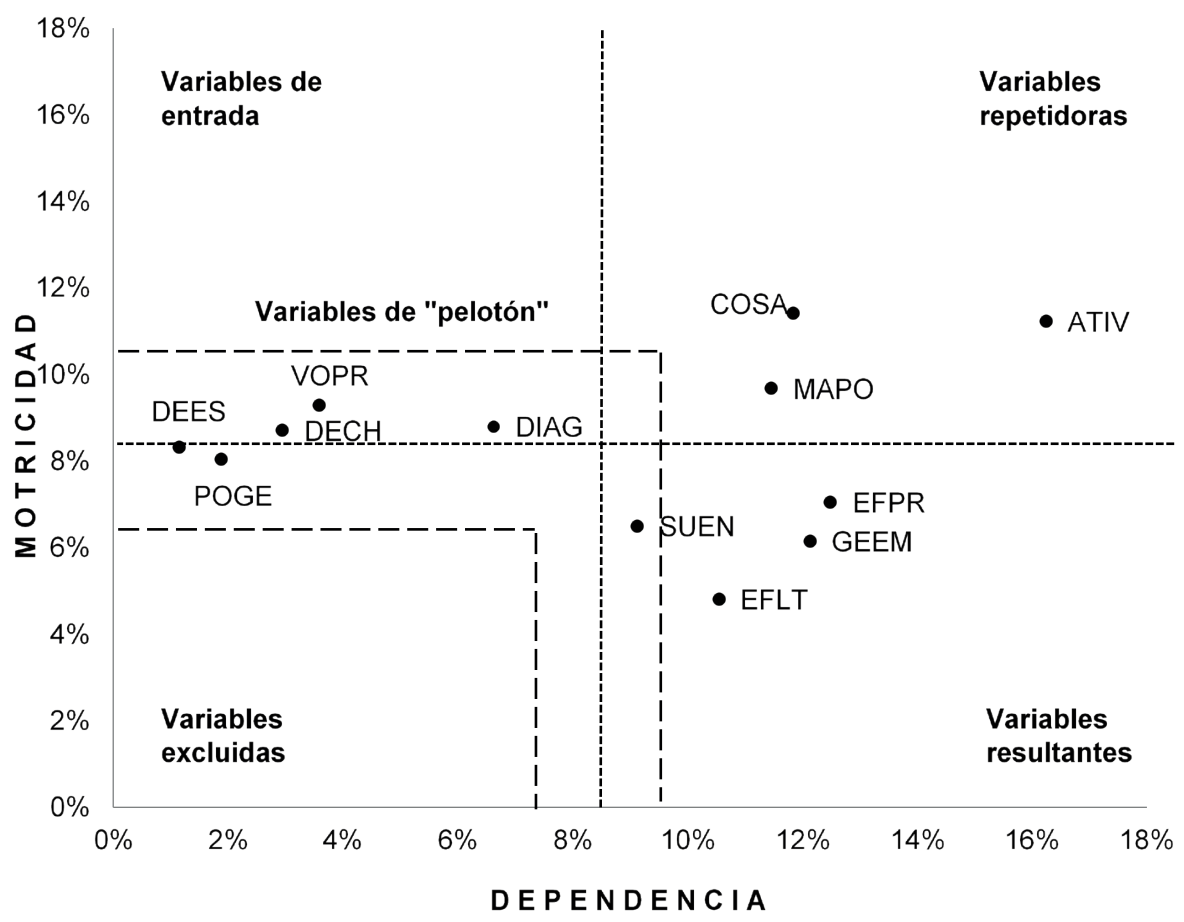

Fuente: Elaboración propia

Las variables clave, ubicadas en el cuadrante superior derecho, son aquellas que presentan mayor puntaje consensuado de motricidad y dependencia; es decir, las más sensibles para todo el sistema. Las variables por debajo del puntaje promedio de dependencia (cuadrantes de la izquierda) se consolidaron exclusivamente en la zona de las llamadas variables de "pelotón" (Godet,
2007); coincidentemente, el resto de las variables exógenas. La no presencia de variables en el cuadrante denominado de variables de "entrada", significa que no existen variables dominantes o de alta motricidad y poca dependencia. Todas las variables endógenas al sistema se ubicaron en la zona propia de las variables "resultantes", caracterizadas por tener alta dependencia pero baja motricidad para el sistema. 


\section{2 ¿Cómo gestionar el sistema? Análisis de las variables clave}

El atractivo de inversión (ATIV) quedó confirmado como la variable más importante para el sistema, siendo la más influyente y dependiente al mismo tiempo. Esto evidencia que la minería se confirma como una industria intensiva en capital. Para el año 2021 se espera en Perú la construcción de 5 proyectos mineros que representan una inversión comprometida de casi 3400 millones de dólares. Estas inversiones permiten prever que la minería será uno de los sectores con mayor recuperación para los años post pandemia, con un crecimiento de $14,4 \%$ del PBI minero (Minem, 2020). El Perú mantiene ventajas competitivas sobre otros países que fortalecen el atractivo de inversión ya que cuenta con mano de obra altamente capacitada y de bajo costo, así como con un sobredimensionamiento energético. Ambos aspectos se reflejan en un costo de producción más competitivo del mercado (Belapatiño et al, 2020).

Sin embargo, estas ventajas no son sostenibles en el tiempo, por lo cual se debe trabajar sobre planes de desarrollo nacional donde la minería sea la industria pilar; de forma que la inversión tenga impactos sobre toda la economía. Los conflictos socioambientales (COSA) afectan la inversión en exploraciones mineras, a pesar del gran potencial geológico (POGE) (Sociedad de Comercio Exterior de Perú [Comex Perú], 2020). Por esta razón, se han empezado a tomar iniciativas para impulsar el atractivo de inversión (ATIV), como la 'Guía de Inversión Minera en el Perú 2020-2021' y el 'Plan Estratégico y Portafolio 2020-2021' que informan a los inversionistas sobre la actualidad minera y los planes de desarrollo de nuevos proyectos en el país. No obstante, las iniciativas no tendrán efectividad si es que no son acompañadas con un plan de comunicación efectiva a las poblaciones involucradas. El gobierno no puede desentenderse y debe participar activamente en las relaciones entre las empresas privadas y las comunidades, demostrando competencia en la relación de influencia entre los conflictos socioambientales (COSA) y el manejo político (MAPO); la tercera variable identificada como clave en el análisis estructural.

En este contexto, el gobierno y las empresas mineras deben trabajar de la mano para poder comunicar a las comunidades que la actividad minera es una oportunidad de desarrollo evidenciada en el tiempo. Según un estudio del Instituto Peruano de Economía (IPE), entre los años 2007 y 2017, 30 mil personas salieron de la pobreza y se duplicó el ingreso per cápita en la zona de influencia operativa de la mina Antamina, al norte de la ciudad de Lima (Instituto de Ingenieros de Minas del Perú [IIMP], 2020). Sin embargo, las comunidades de hoy no tienen visibilidad de los beneficios propiciados por la minería. Según una investigación de Aurum, Consultoría y Mercado, el $44 \%$ de ciudadanos de zona urbana de la ciudad norteña de Cajamarca, desconocen el canon minero a pesar de ser la provincia que más transferencias mineras ha recibido en los últimos años (Energiminas, 2020a).

En efecto, el canon minero debería ser impulsor del crecimiento de las comunidades aledañas a los proyectos mineros. Según un estudio de la Iniciativa para la Transparencia en las Industrias Extractivas (EITI), el 
$55 \%$ del presupuesto de inversiones de los municipios de la región sureña de Arequipa proviene del canon y regalías mineras (Energiminas, 2020b). Por ello, los expertos alcanzaron consenso en que la solución más eficiente para aprovechar el potencial minero que tiene el Perú es la proclamación de una política minera que se encargue de incentivar el desarrollo continuo de las actividades de este sector; así como también la exploración minera dado el potencial geológico (POGE) peruano, la optimización de excesivos procesos burocráticos y el aseguramiento de una participación activa en las mesas de diálogo con las comunidades afectadas.

\section{3 ¿Cómo gestionar el sistema? Análisis del resto de variables subordinadas}

En cuanto a las cuatro variables endógenas, éstas se ubicaron en la zona de variables "resultantes" para el sistema -con alta dependencia y baja motricidad- debido a que se subordinan a factores externos. El suministro de energía eléctrica (SUEN) aumentará debido al desarrollo de 19 proyectos y ampliaciones mineras en todo Perú (Osinergmin, 2019). En ese contexto, el país mantiene una ventaja competitiva global con relación al costo de energía gracias a la presencia de plantas de energía hidráulica y de gas natural. La energía hidráulica aporta el $44 \%$, la energía proveniente del gas natural de Camisea, el $45 \%$; y el $11 \%$ restante es por energía eólica, solar y diésel (ContentLab, 2020).

La razón de la competitividad del costo de energía es el sobredimensionamiento del sistema eléctrico, que impacta en la eficiencia de la producción (EFPR) que, en su momento, se proyectaba para cubrir una amplia cartera de proyectos mineros. Lamentablemente, muchos han sido retrasados por los conflictos socio-ambientales (COSA). Esta ventaja no será sostenible en el tiempo debido al crecimiento de la demanda energética mencionada, por lo que la nueva tendencia mundial de migrar hacia fuentes de energía renovable se pronuncia cada vez más. Incluso, el objetivo de reducir las emisiones de $\mathrm{CO}_{2}$ al ambiente, ha originado que países con los que Perú compite -como Australiaempleen energía eléctrica renovable para sus equipos de extracción y transporte (The Warren Center, 2020).

La minería peruana debe alinearse con estas políticas globales, ya que tiene el potencial de desarrollar energía renovable eólica y solar. En la actualidad, Cerro Verde, la quinta mina de cobre peruano más grande del mundo (Statista, 2019), está incentivando un proyecto de una planta de energía solar de $200 \mathrm{MW}$. Sin embargo, los procesos de licitación -legales y regulatorios- dificultan su avance exponiendo las dificultades que se tienen en el manejo político (MAPO) peruano (La República, 2020). Algunos investigadores mencionan que el Perú podría contar con un suministro $100 \%$ solar para el año 2030 (Haas et al, 2020), pero esto fue cuestionado por los expertos participantes en la investigación. Éstos coincidieron en que la energía solar debe ser complementaria, ya que una mina requiere de suministro energético las 24 horas y tanto la energía eólica como la solar son estacionarias. Además, las baterías para almacenamiento de estas últimas formas de energía resultan una inversión muy elevada cuando se comparan al costo de la energía eléctrica. 
La minería es una actividad intensiva en capital aunque no en mano de obra. Por ello, la variable generación de empleo (GEEM) pierde relevancia. No obstante, la actividad minera crea encadenamientos productivos que enlazan la misma logística del transporte, la eficiencia de producción, el personal de ventas y las relaciones internacionales con los países a los que se exporta. Por esta razón, se valida la premisa que, por cada empleo en minería, se generarían 6,25 empleos indirectos en el resto de la economía (Minem, 2020). Sin embargo, el valor que otorga una comunidad a un proyecto minero no se encuentra solo en la posibilidad de obtener un trabajo, sino en el mejoramiento de las condiciones de vida que alivian las tensiones de los conflictos socio-ambientales (COSA).

Por su parte, al encontrarnos en una era de transformación digital de las empresas, el sector minero se ha visto afectado y, como consecuencia, busca reducir la presencia de mano de obra en las minas (Rumbo Minero, 2020). Esto podría resultar contraproducente y elevaría el nivel de conflictos socioambientales con la comunidad local debido a que una menor cantidad de personas de la región serían contratadas; percibiéndose los intereses de las mineras ajenos a los de los pobladores.

La contraparte indica que, al contar con maquinaria o procesos digitalizados, se requerirá personal capacitado técnicamente. De esta forma, una parte de la población se vería beneficiada educativamente. Actualmente, en las regiones peruanas sureñas de Ica y Moquegua, se está explorando la idea de camiones autónomos en los proyectos de Minajusta y Quellaveco; ya que en países como Canadá y Australia, éstos son una realidad capaz de elevar la competitividad (Latam Mining, 2020).
En cuanto a la volatilidad del precio del cobre (VOPR) en el mercado de commodities, éste ejerce presión sobre la eficiencia de la producción (EFPR). Esta última, además, es fuertemente influenciada por los efectos de la demanda y oferta del cobre; y además, como coincidieron los expertos en el análisis estructural con Henckens y Worell (2020), podría incrementarse en 10 veces su valor actual para el año 2050. Es importante entender los ciclos del precio y reconocer en qué fase del mercado se encuentran, analizando los históricos y proyectando sus tendencias.

De acuerdo a la estatal Comisión Chilena del Cobre (Cochilco), se proyecta que para el año 2021 el precio se mantendrá en trayectoria alcista, alcanzando un promedio de US\$2,85 por libra; superior al precio promedio que mantuvo en el año 2020 de US\$ 2,62 por libra (Portal Minero, 2020). Estos cambios impactan directamente en las empresas, ya que las operaciones deben cumplir con los plazos establecidos en los contratos comerciales firmados por adelantado.

Se hace necesario, por lo tanto, mantener estrategias que favorezcan la eficiencia de los costos para los periodos en los cuales el precio decrece; ya que si se tuviera un cambio brusco en el precio, la reducción de los costos de operación no sería súbita y permitiría escudarse por los bajos márgenes de ganancia que se obtendrían. Si se proyectan tendencias hacia el alza o periodos de auge, se generarían elevados márgenes de ganancia haciendo que no sea muy necesario preocuparse por una eficiencia estricta en costos.

El resto de las variables no presentan una influencia importante para el sistema minero del cobre peruano. Dentro de este grupo, se encuentran las 
variables comerciales como la demanda de China $(\mathrm{DECH})$ y la demanda de economía sostenible (DEES). Ello implica que la inestabilidad del sistema no se explicaría por la demanda del cobre. Los expertos coincidieron en que, si bien es cierto que la demanda de China $(\mathrm{DECH})$ consume el $50 \%$ de la producción mundial de cobre (Belapatiño et al, 2020); el país asiático está llegando a un nivel de madurez avanzado de urbanización que podría descentralizar la demanda hacia otros países que se encuentren aún en tal carrera, como India.

Según un estudio del Departamento de Industria, Innovación y Ciencia del gobierno australiano (Gestión, 2017), la población urbana de India podría incrementarse en un $46 \%$ para el año 2035, alcanzando los 642 millones de habitantes y proyectándose que la demanda de cobre llegaría a ser de 2,8 millones de toneladas métricas para ese año en un escenario conservador (basándonos en el crecimiento de Brasil, Argentina y México) o de 13,5 millones de toneladas métricas en un escenario más agresivo (basándonos en el crecimiento de China).

Adicionalmente, los expertos coincidieron en la importancia de la demanda de la economía sostenible (DEES), concordando en que uno de los principales mercados que impulsaría la transición sería el de los vehículos eléctricos que, según los reportes de la Asociación Internacional del Cobre (ICA, 2017) tuvo un crecimiento de 9,5 veces para el 2017 (alcanzando 1,74 millones de toneladas métricas). Cabe resaltar que las principales fundiciones del mundo -que se localizan en Chinaprocuran asegurar el suministro de energía por adelantado. De esta forma, la demanda seguirá dependiendo de
China a menos que se empiece a relocalizar la instalación de las mismas (Gestión, 2020).

Finalmente, aunque no por ello menos relevante, al evaluar la importancia de la disponibilidad del agua (DIAG) y su relación con las otras variables, se determinó que desencadena conflictos de orden socio-ambiental (COSA) en la mayoría de los casos. Esto se debe a que muchas comunidades protegen sus reservas de agua, complejizando el manejo de relaciones que las empresas mineras deben tener con la comunidad. Si bien el agua es abundante al norte de Perú, en las regiones sureñas, escasea. Esta problemática, también advertida por los expertos participantes, confirma lo ya señalado por González et al, (2019) acerca de los serios problemas de escasez del líquido elemento que se prevén en el sur de Perú para los próximos diez años.

\section{Conclusiones}

La evidencia obtenida a partir de esta investigación identificó como variables clave para la industria del cobre en Perú al atractivo por la inversión, a los conflictos socioambientales y al manejo político; todas ellas exógenas. El resto de las variables externas del sistema no presentan un sesgo motriz o dependiente, por lo cual su impacto terminaría siendo incierto para la industria cuprífera peruana.

Aspectos endógenos (internos) al sistema como la eficiencia productiva, la logística del transporte, la generación de empleos y el suministro de energía; se caracterizan como intrínsecamente dependientes de la evolución de las variables clave anteriormente mencionadas. En consecuencia, la inestabilidad del sistema estudiado se 
explicaría por la presencia de dichas variables clave, dado su carácter altamente influyente y dependiente de forma simultánea. Asimismo, el análisis estructural no advierte la presencia de variables exclusivamente motrices ni dependientes para el sistema.

Finalmente, un adecuado manejo de este importante sector económico de la industria peruana involucraría a tres agentes directamente responsables de la conducción de las variables clave: el Estado, el sector privado y las comunidades afectadas por la extracción minera. La reflexión colectiva producto del análisis estructural prevé que solo un diálogo abierto y efectivo, en aras de la concurrencia de los intereses de todas las partes involucradas, sería determinante para la adecuada reconducción del atractivo de inversión minera, de los conflictos socioambientales y del manejo político del sector. Además, por inercia y repercusión consecutiva y estructural, todo el sistema se vería beneficiado. Por ello, focalizar los planes de acción sobre la base de dichas variables clave constituye el primer paso para la gestión adecuada de posibles escenarios futuros vinculados a la evolución del sector minero del cobre en Perú y toda la región; y a sus repercusiones políticas, sociales, ambientales y económicas a mediano y largo plazo.

\section{Referencias bibliográficas}

Alvarado, J. A. (2018). Impactos económicos y sociales de las políticas nacionales mineras en Ecuador (2000-2006). Revista De Ciencias Sociales, 23(4), 53-64. https://doi. org/10.31876/rcs.v23i4.25137

Asociación Internacional de Cobre [ICA]. (2017). The electric vehicle market and copper demand. IDTechEx.
https://cutt.ly/2kVL53p

Asociación Internacional de Cobre [ICA]. (2019). 2019 Annual Report. https:// cutt.ly/fkVZpf7

Belapatiño, V., Crispin, Y., y Villanueva, A. (2020). Perú. Sector Minero: evolución reciente y perspectivas de corto plazo. BBVA Research. https:// cutt.ly/DkVLjGo

Cárcamo, G. M. (2018). El efecto del precio de los minerales sobre la estructura de capital de las empresas mineras en el Perú (2006 - 2016) [tesis de licenciatura en Economía, Universidad de Lima]. Repositorio Institucional - Universidad de Lima.

ContentLab. (2020). Minería verde: 5 claves para tener energía sostenible. https://cutt.ly/tkVNytN

Cornejo, R. (2018). Las cadenas logísticas mineras en el Perú: oportunidades para una explotación más sostenible de los recursos naturales. CEPAL, Comisión Económica para América Latina y el Caribe. https://cutt.ly/9kVZPa7

De Echave, J. (2014). La minería en el sur andino: los casos de Cuzco y Apurímac. https://cutt.ly/YkVCDaC

De Echave, J. (2016). La minería ilegal en Perú Entre la informalidad y el delito. Nueva Sociedad. https://cutt. Iy/ZkVCGhc

Dirección General de Promoción y Sostenibilidad Minera [DGPSM]. (2020). Cartera de Proyectos de Construcción de Mina 2019. Ministerio de Energía y Minas del Perú. https://cutt.ly/GkVLzOQ

El Peruano. (febrero de 2019). Inversiones mineras sumaron US\$4,947 millones en el 2018. https://cutt.ly/5kVCrCZ

Energiminas. (2020a). Grupo Estrategia: 
$44.3 \%$ de ciudadanos de zona urbana de provincia de Cajamarca desconoce el canon minero. https:// cutt.ly/8kVBYwL

Energiminas. (2020b). 55\% de presupuesto de inversión de municipios de Arequipa es cubierto por canon y regalías mineras. https:// cutt.ly/VkVBLMM

Gahona-Flores, O. (2020). Gestión de Proveedores en la Cadena de Suministro de la Minería del Cobre en Chile. Revista Venezolana De Gerencia, 25(92), 1671-1683. https:// doi.org/10.37960/rvg.v25i92.34288

García, M., y Suárez, M. (2013). El método Delphi para la consulta a expertos en la investigación científica. Revista Cubana de Salud Pública, 39(2), 253-267. https://www. redalyc.org/pdf/214/21430556007. pdf

García, M., y Pantigoso, P. (2020). Peru's mining \& metals investment guide 2020 / 2021. Ministerio de Relaciones Exteriores del Perú. https://cutt.ly/VkVJFoM

Gestión. (2017) ¿Es la India la próxima China?, se pregunta estudio australiano. https://cutt.ly/hkVNDs2

Gestión. (2020). BBVA: producción minera acelerará en 0.6 puntos porcentuales el crecimiento del PBI en 2020. https://cutt.ly/VkVCh7X

Godet, M. (2007). Prospectiva Estratégica: problemas $y$ métodos. Prospektiker. http://www. prospektiker.es/prospectiva/cajaherramientas-2007.pdf

Godet, M., y Durance, P. (2011). La prospectiva estratégica para las empresas y los territorios. Unesco, Organización de las Naciones Unidas para la Educación, la Ciencia y la Cultura. https://cutt.ly/okVVJAG
Gomero, N. (2017). Volatilidad de los commodities mineros y su incidencia en la economía nacional. Revista Quipukamayoc, 25(48), 91-100. https://doi.org/10.15381/quipu. v25i48.13999

Gonzales, F., Raval, S., Taplin, R., Timms, W., y Hitch, M. (2019). Evaluation of Impact of Potential Extreme Rainfall Events on Mining in Peru. Natural Resources Research, 28, 393-408. https://doi.org/10.1007/ s11053-018-9396-1

Haas, J., Moreno-Leiva, S., Junne, T., Chen, P., Pamparana, G., Nowak, W., Kracht, W., y Ortiz, J. (2020). Copper mining: $100 \%$ solar electricity by 2030? Applied Energy, 262, 114506. https://doi.org/10.1016/j. apenergy.2020.114506

Henckens, M., y Worell, E. (2020). Reviewing the availability of copper and nickel for future generations. The balance between production growth, sustainability and recycling rates. Journal of Cleaner Production, 264, 121460. https://doi.org/10.1016/i. jclepro.2020.121460

Instituto de Ingenieros de Minas del Perú [IIMP]. (2020). Antamina: Estudio del IPE revela que unas 30,000 personas de su área de influencia salieron de la pobreza. https://cutt.ly/okVBaDS

Instituto Geológico, Minero y Metalúrgico [Ingemmet]. (2017). Estimación del potencial minero metálico del Perú y su contribución económica al estado, acumulado al 2050. Dirección de Recursos Minerales y Energéticos. https://www.gob.pe/ingemmet

Instituto Nacional de Estadística e Informática [INEI]. (2020a). Comportamiento de los indicadores de mercado laboral a Nivel Nacional. https://cutt.ly/gkVCmF8

Instituto Nacional de Estadística e 
Fernando; Chirinos-Cuadros, Carlos Ricardo

Minería del cobre en Perú: análisis de las variables exógenas y endógenas para gestionar su desarrollo

Informática [INEI]. (2020b). Población con al menos una necesidad básica insatisfecha, según departamento. https://cutt.ly/ZkVC7wz

Lalama, R. A., y Bravo, A. (2017). Capitalismo social: un vistazo a resultados macroeconómicos de Ecuador, Perú y Colombia. RETOS. Revista de Ciencias de la Administración y Economía, 7(13), 91. https://bit.ly/2PEwZNV

Lalama, R. A., y Bravo, A. (2019). América Latina y los objetivos de desarrollo sostenible: Análisis de su viabilidad. Revista De Ciencias Sociales, 25, 12-24. https://doi.org/10.31876/rcs. v25i1.29591

La República. (2020). Proyectos de energía renovable en minería se frenan por demora de permisos. https://cutt.ly/4kVMob7

Latam Mining. (2020). Perú: Ferreyros iniciará proyecto $100 \%$ autónomo en Quellaveco. https://cutt.ly/PkVNm31

León, J. (2019). Determinantes económicos y sociopolíticos de los conflictos socioambientales en el Perú. Revista de Investigaciones Altoandinas, 21(2), 122-138. http:// dx.doi.org/10.18271/ria.2019.456

Lominchar, J. (2020). Visión del cambio Covid-19: impacto en la fuerza de trabajo y profesionales autónomos. Revista Venezolana De Gerencia, 25(4), 12-31. https:// produccioncientificaluz.org/index. php/rvg/article/view/35213

Ministerio de Energía y Minas [Minem]. (2019). Minem: Casi el 10\% del PBI y el $61 \%$ de las exportaciones del 2018 fueron producto de la minería. https:// cutt.ly/okVZV06

Ministerio de Energía y Minas [Minem]. (2020). Informe del empleo minero 2019: Panorama y tendencias en el Perú. Dirección General de Promoción y Sostenibilidad Minera. https://minem.gob.pe/ publicacion. php?idSector $=1$ \&idPublicacion $=594$

Nugra Betancourth, M. A., Illescas Espinoza, W. H., Cuadros García, P. A., y Valdivia Ramos, R. A. (2021). Turismo minero en Yanacocha: Una alternativa de desarrollo para la región de Cajamarca-Perú. Revista De Ciencias Sociales, 27(1), 278289. https://produccioncientificaluz. org/index.php/rcs/article/view/35313

Organismo Supervisor de la Inversión en Energía y Minería [Osinergmin]. (2017). La industria de la minería en el Perú: 20 años de contribución al crecimiento y desarrollo económico en el país. https://cutt.ly/GkVXQ5q

Organismo Supervisor de la Inversión en Energía y Minería [Osinergmin]. (2019). Relación actual y futura entre el sector minero y el sector eléctrico. Gerencia de Políticas y Análisis Económico. https://cutt.ly/QkVB7k3

Portal Minero. (2020). Cochilco eleva proyección del precio del cobre a US\$2,62 la libra para 2020. https:// cutt.ly/rkVNUGi

Rivera, L. (2020). Semáforo Minero: Coronavirus y transformación digital en minería. Instituto de Ingenieros de Minas del Perú. https://cutt. ly/5kVVUQV

Rodrigo-Cano, D., Josep Picó, M., y Dimuro, G. (2019). Los Objetivos de Desarrollo Sostenible como marco para la acción y la intervención social y ambiental. RETOS, Revista de Ciencias de Administración y Economía, 9(17), 25-36. https://doi. org/10.17163/ret.n17.2019.02

Rojas de la Puente, E. E., Rimarachín Chavez, F. L., Oblitas Cruz, J., y Castro Silupu, W. (2019). Control de proyectos de Responsabilidad Social 
pp. 784-801

Revista Venezolana de Gerencia, Año 26 No. 94, 2021

empresarial: Estudio en empresas mineras. Revista Venezolana De Gerencia, 24(87), 684-700. https://doi.org/10.37960/revista. v24i87.24632

Rumbo Minero. (2020). La tecnología y los robots autónomos: el futuro de la minería subterránea. https://cutt.ly/ tkVVWUe

Ruiz-Ruiz, M. F. (2017). La prospectiva francesa como estrategia de planeación universitaria: evaluación de la aceptabilidad de su aplicación en una facultad de ingeniería [Tesis de doctorado, Pontificia Universidad Católica del Perú]. http://tesis.pucp.edu.pe/repositorio/ handle/20.500.12404/7929

Schwarz, M. (2018). Inteligencia artificial en la industria minera. Energiminas. https://www.energiminas.com/ wp-content/uploads/2018/11/ Energiminas-67.pdf

Servicio Geológico de los Estados Unidos [USGS]. (2020). Mineral commodity Summaries 2020. U.S. Geological Survey. https://doi.

\section{org/10.3133/mcs2020}

Sociedad de Comercio Exterior del Perú [Comex Perú]. (2020). La importancia de solucionar los conflictos sociales en el Perú. https://cutt.ly/8kVV4ug

Sociedad Nacional de Minería, Petróleo y Energía [SNMPE]. (2020). Sector minero peruano captó inversiones por US\$ 25,191 millones en quinquenio 2015-2019. https://cutt.ly/pkVX7wq

Sociedad Nacional de Minería, Petróleo y Energía [SNMPE]. (2021) ¿Qué es el Canon Minero? https://bit. ly/3c8kdyy

Statista. (2019). Ranking de las mayores minas de cobre a nivel mundial en 2019, por capacidad de producción. https://bit.ly/3bh8u1t

The Warren Center. (2020). Zero Emission Copper Mine of the Future. https://copper.com.au/wordpress/ wp-content/uploads/Zero-EmissionsCopper-Mine-of-the-Future-Report. pdf 